\title{
Assessing the Validity of the Quiet Standing Inverted Pendulum Model
}

\author{
Chen-Wen Yen ${ }^{1,2}$, Chih-Yuan Hong ${ }^{1}$ and Jia-Li Sung ${ }^{1}$ \\ ${ }^{1}$ Department of Mechanical and Electro-mechanical Engineering, National Sun Yat-Sen University, Taiwan \\ ${ }^{2}$ Department of Physical Therapy, Kaohsiung Medical University, Kaohsiung, Taiwan
}

\begin{abstract}
This study develops a feature to quantify the degree of validity of the IP model for quiet standing human body. By denoting the anterior-posterior component of the ground reaction force (GRF) as GRF $F_{x}$ and defining COP error as the difference between the displacements of the center of pressure (COP) and the vertical projection of center of mass (COM), the proposed feature is chosen as the correlation coefficient between GRF $F_{x}$ and COP error signals. The rationale behind such a design is the property of the IP model which states that GRF $F_{x}$ is proportional to COP error. The efficacy of the proposed feature is demonstrated by comparing its differences between two age groups in quiet standing. The experimental results show that the IP model can more accurately predict the correlation strength between COP error and GRF $F_{x}$ of the older group than the younger group. This seems to suggest that the older group behaves more like an IP than the younger group.
\end{abstract}

Keywords: inverted pendulum, postural stability, quiet standing, force platform

\section{Introduction}

With center of pressure (COP) as the controlling variable and center of mass (COM) as the controlled variable of the balance control system, the quiet standing human body has been modelled as an inverted pendulum (IP) which is stabilized by the ankle joint [1-2]. An important property of such a one degree-offreedom single joint IP model is that the COM's horizontal acceleration is proportional to the difference between COP and the vertical projection of COM. This difference was considered to be the balance control system error signal that induces COM's horizontal acceleration [1] and will be referred to as the "COP error" in the remaining part of this manuscript.

The validity of the IP model has been supported by experimental results which show that the COP error is indeed highly correlated with the horizontal acceleration of the COM [2-5]. However, several studies have challenged the validity of the single joint IP model by showing that multiple body joints contribute actively and consistently to quiet human stance [6-10]. In particular, these results seem to indicate that human body may perform like a dynamic system with multiple degrees-of-freedom (DOFs). As a result, the single DOF IP is an incomplete model for human standing.

This work proposes an index to characterize the degree of validity of IP model by quantifying the correlation strength between the COP error and horizontal COM acceleration in the anterior-posterior (AP) direction. By addressing the interactions between the validity of IP model, the co-variation of joint motions, the postural stability and the proposed feature, it is hoped that the results of this work can increase our understanding on the coordination patterns of human body segments during quiet standing. 


\section{Method}

\subsection{Participants}

The volunteers participated in this study consisted of two age groups - an older age group $(68.7 \pm 2.96$ years, range $65-73$ years; BMI $23.9 \pm 4.13 \mathrm{~kg} / \mathrm{m} 2)$ and a younger age group $(20.1 \pm 1.29$ years, range $18-24$ years; BMI $22.5 \pm 3.21 \mathrm{~kg} / \mathrm{m} 2$ ). Each of these two groups consists of 10 male and 10 female healthy adults. Based on a self-report and a physical examination, none of the subjects had a pathological condition that would comprise their postural performance. All experimental procedures were approved by the Institutional Review Board of the Kaohsiung Medical University Chung-Ho Memorial Hospital, Kaohsiung, Taiwan.

\subsection{Measurements}

Each subject was tested for two days. Two experimental sessions were performed both days. Each session included three $80 \mathrm{~s}$ eyes-open-closed trials. In the first $40 \mathrm{~s}$ of these trials, the subjects were asked to look straight ahead at a visual reference and stand quietly (with arms at the side) in a comfortable stance near the center of the force platform. By trying to maintain the same posture, the subjects closed their eyes in the remaining $40 \mathrm{~s}$ of the trial. The data collected from 5 to $35 \mathrm{~s}$ and 45 to $75 \mathrm{~s}$ of the trials were used for this study. Trials and sessions were separated by approximately one and five minutes of rest, respectively.

The force measurement system consisted of one force platform (9286AA, Kistler) connected to a PC-based data acquisition system. With a sampling rate of $512 \mathrm{~Hz}$, the force platform measurement results were sent to a 14-bit ana log-to-digital data acquisition card (USB-6009, National Instruments) connected to a desktop PC. The data processing software was a custom-developed program written in LabVIEW (National Instruments). The signals were filtered by a sixth-order Butterworth filter with a cutoff frequency of $5 \mathrm{~Hz}$.

\subsection{Measurements}

In the AP direction, the IP model can be represented by the following equation [1]:

$$
x(t)-p_{x}(t)=e_{x}(t)=I_{a} \ddot{x}(t) / m g h=I_{a} \mathrm{GRF}_{x}(t) /\left(m^{2} g h\right)
$$

where $x$ and $p_{x}$ are the COM and COP displacements with respect to the ankle joint, respectively, $e_{x}$ is called the COP error, $I_{a}$ is the moment of inertia of the total body about the ankle joint in the sagittal plane, $m$ is the mass of the body (excluding the feet), $g$ is the gravitational constant, $h$ is the COM height above the ankle joint and $\mathrm{GRF}_{x}$ is the AP component of the ground reaction force (GRF).

Since $\mathrm{GRF}_{x}$ is proportional to the AP component of the COM's acceleration, a possible approach to confirm the validity of the IP model is to compute the correlation coefficient between $e_{x}$ and $\mathrm{GRF}_{x}$. To determine this coefficient, with COP signal $p_{x}$ and GRF signal $\mathrm{GRF}_{x}$ obtained from the force platform, this work uses a lowpass filtering technique to estimate COM signal $x(t)$ [11-12]. Specifically, this method assumes that the frequency domain transfer function from COP to COM can be represented by:

$$
X(\omega) / P_{x}(\omega)=\omega_{0}^{2} /\left(\omega_{0}^{2}+\omega^{2}\right)
$$

where $\omega$ is the frequency variable $(\mathrm{rad} / \mathrm{sec}), X(\omega)$ and $P_{x}(\omega)$ are the Fourier transforms of $x(t)$ and $p_{x}(t)$, respectively and $\omega_{0}$ is the natural body frequency. Once $\omega_{0}$ is known, one can readily use the following procedure to determine the desired correlation coefficient.

1. Determine COM spectrum $X(\omega)$ from equation (2).

2. Taking the inverse transform of the resulting $X(\omega)$ to yield $x(t)$.

3. Compute the COP error signal $e_{x}$ from equation (1).

4. Determine correlation coefficient between $e_{x}$ and $\mathrm{GRF}_{x}$.

The value of the correlation coefficient between $e_{x}$ and $\mathrm{GRF}_{x}$ should be close to 1 if IP is a valid model for quiet standing posture. Since $\omega_{0}$ was estimated to range from 2.8 to $3.2 \mathrm{rad} / \mathrm{sec}$ [11], this study progressively varied the value of $\omega_{0}$ from $1.0 \mathrm{rad} / \mathrm{sec}$ to $5.0 \mathrm{rad} / \mathrm{sec}$ with an increment of $0.1 \mathrm{rad} / \mathrm{sec}$. By using the above procedure to determine the correlation coefficients between $e_{x}$ and $\mathrm{GRF}_{x}$ for all tested $\omega_{0}$ values, this work chose 
the largest correlation coefficient value as the proposed feature. This feature will be referred to as the IP validity index (IPVI) hereafter.

For comparison, this work also used a COP velocity feature to assess postural stability. Traditionally, the COP velocity can be represented by the mean velocity (MV), mean velocity in the medial-lateral (ML) direction $\left(\mathrm{MV}_{\mathrm{ML}}\right)$ and mean velocity in the AP direction $\left(\mathrm{MV}_{\mathrm{AP}}\right)$. This study chose $\mathrm{MV}_{\mathrm{AP}}$ as the benchmark reference since it was considered one of the most sensitive COP measures for postural control assessment [13-15].

\subsection{Data Analyses}

Data analyses were performed to assess the differences between the younger and older groups in the eyes open condition. The first part is to compare the means of the tested features of the older group to those of the younger group by using independent Student's $t$-test. For the sake of reliability, this study used the average of the twelve trials ( 3 measurements/session $\times 4$ sessions) as the sample value.

The second part of the data analysis work tries to evaluate the association between the proposed feature and postural steadiness quantified by $\mathrm{MV}_{\mathrm{AP}}$. Specifically, for a given tested subject, the 30 second process of every experimental trial was first divided into thirty non-overlapping one second windows. This generated 360 windows of data for each subject. Based on the magnitude of IPVI, these windows were divided into two halves. To use the independent Student's $t$-test to assess the difference between the $\mathrm{MV}_{\mathrm{AP}}$ means of the larger and smaller halves of the IPVI values, this work chose the average $\mathrm{MV}_{\mathrm{AP}}$ values of each tested subject's larger and smaller halves of the IPVI values as the sample.

\section{Results}

As shown in Table 1, in agreement with previous studies, the mean value of the MVAP of the older group is significantly larger than that of the younger ground. Also in agreement with the previous studies, the results of Table 1 show that the mean values of the proposed IPVI feature are larger than 0.9 for both age groups. These results of IPVI demonstrate that, in the AP direction, the COP error is indeed highly correlated with the GRF and thus support the validity of the IP model. However, Table 1 also shows that the mean IPVI value of the older group is significantly larger than that of the younger group. This seems to suggest that the IP model can more accurately represent the quiet standing human body of the older group than the younger group.

TABLE I: Mean, standard deviations and the $p$-values of the COP feature $M V_{\mathrm{AP}}$ and the proposed feature IPVI

\begin{tabular}{|c|c|c|c|}
\hline \multirow{2}{*}{ Features } & \multicolumn{2}{|c|}{ Age group } & \multirow{2}{*}{$p$-values } \\
\hline & Younger & Older & \\
\hline $\mathrm{MV}_{\mathrm{AP}}$ & $5.45 \pm 1.06$ & $7.28 \pm 2.0$ & $9.52 \times 10^{-4}$ \\
\hline IPVI & $0.922 \pm 0.021$ & $0.958 \pm 0.015$ & $3.05 \times 10^{-1}$ \\
\hline
\end{tabular}

Values of the features are mean \pm standard deviation

Units of features are as follows: $\mathrm{mm} / \mathrm{s}\left(\mathrm{MV}_{\mathrm{AP}}\right)$, dimensionless (IPVI )

Table 2 summarizes the comparative results of the MVAP feature associated with the larger and smaller halves of the IPVI values. As shown by Table 2, for both age groups, the mean MVAP of the larger half of the IPVI values is significantly larger than that of the smaller half of the IPVI values.

TABLE II: Mean, standard deviations and the $p$-values of feature $\mathrm{MV}_{\mathrm{AP}}$ for the two halves of the IPVI feature values

\begin{tabular}{llll}
\hline \hline \multirow{2}{*}{ Age group } & \multicolumn{2}{c}{ IPVI values } & \multirow{2}{*}{-values } \\
\cline { 2 - 4 } & The larger half & The smaller half & $8.67 \times 10^{-9}$ \\
\hline Younger & $6.83 \pm 1.30$ & $4.37 \pm 0.75$ & $3.21 \times 10^{-6}$ \\
\hline Older & $8.87 \pm 1.93$ & $5.97 \pm 1.39$ & \\
\hline \hline
\end{tabular}

Values of the features are mean \pm standard deviation

\section{Discussions}

By showing the COP error and GRF signals are indeed highly correlated in the AP direction, the IPVI results of Table 1 support the validity of the IP model. However, since the mean IPVI value of the older group in 
significantly larger than that of the younger group, the degree of validity of the IP model changes with age. This seems to imply that the older group behaves more like an IP than the younger group.

For both age groups, the results of Table 2 indicate that the mean $\mathrm{MV}_{\mathrm{AP}}$ of the larger half of the IPVI values is significantly larger than that of the smaller half of the IPVI values. This result and the results of Table 1 show that larger IPVI is associated with larger $\mathrm{MV}_{\mathrm{AP}}$. As reported by many previous studies, large $\mathrm{MV}_{\mathrm{AP}}$ represents poor postural instability, therefore it is reasonable to assume that IPVI can also be used to assess postural stability.

A fundamental goal of coordinating body joints is to keep COM within safe limits of the support surface. To achieve this goal, the IP model replies only on ankle motion. In questioning the validity of the IP model, many studies demonstrated the essential roles of the remaining body joints and address the importance of the joint coordination. For example, by using the uncontrolled manifold (UCM) approach to analyze the effect of joint configuration variance on the stability of the COM, Hsu et al. [7] found that all major joints along the longitudinal axis of the body are equally active during quiet standing. By finding the PCA (principal component analys is) components of the variance of lower leg, upper leg and head-arms-trunk angles, Pinter et al. [8] discovered the contributions of upper leg and trunk angle to COM motion. By directly measuring ankle, knee and hip joint kinematics using a vision system, Gunther et al. [9] demonstrated that all leg joints contribute actively to quiet human stance. These results indicate that the quiet human stance is actually a system of multiple degrees of freedom (DOFs). Postural control can therefore be formulated as a problem of motor redundancy which occurs when the DOFs of the motor control system is larger than the number of motor control task [16]. Such redundancy can be resolved through coupling or constraining the DOFs of the motor control system. This is essentially the strategy employed by the IP model since it only uses the ankle joint to control COM.

In instead of limiting the functions of the redundant DOFs, the additional DOFs can be used to improve the performance of the motor control tasks or counteract the influences of unwanted disturbances. For example, experimental results have shown that constraining the redundant DOFs by immobilizing the knees, hips and trunk increased the postural sway [17-18] whereas utilizing such redundancy can compensate the effect of breathing on postural steadiness [19]

The results of this study and previous work suggest that the more the redundant DOFs of the balance control system are put to use, the less likely human body behave like an IP. In this regard, the proposed IPVI feature represents a simple and effective measure that can relate the degree of validity of the IP model with the coordination pattern of the body joints. By monitoring the joint motion with vision systems, a promising future work is to study the interactions between joint coordination pattern, postural stability and the proposed feature.

\section{Conclusion}

By quantifying the correlation strength between the COP error (the difference between COP and COM) and the horizontal component of the ground reaction force $\left(\mathrm{GRF}_{x}\right)$, this work develops a feature to characterize the degree of validity of the inverted pendulum (IP) model for human quiet standing. By comparing the proposed IP validity index (IPVI) values of two age groups, it is found that the IP model can more accurately predict the correlation strength between COP error and $\mathrm{GRF}_{x}$ of the older group than the younger group. Such a result seems to suggest that the older group behaves more like an IP than the younger group. Furthermore, the results of this work and previous studies that demonstrate the active roles of the multiple body joints on posture balance suggest that using the redundant DOFs of the body joints can improve the postural stability while diminishing the validity of the IP model. As a validity index for IP model, the proposed feature can therefore be used to assess the quiet standing coordination patterns of the body segments. 


\section{References}

[1] D. A. W inter, "Hu man balance and posture control during standing and walking," Gait Posture, vol. 3, pp. 193-214, 1995. https://doi.org/10.1016/0966-6362(96)82849-9

[2] D. A. Winter, A. E. Patla, F. Prince, M. Ishac, and K. Gielo-Perc zak, "Stiffness control of balance in quiet standing," $J$ Neurophysiol, vol. 80, pp. 1211-1221, 1998.

[3] D. A. Winter, A. E. Patla, M. Ishac, and W. H. Gage, "Motor mechanisms of balance during quiet standing," $J$ Electromyogr Kinesiol, vol. 13, pp. 49-56, 2003. https://doi.org/10.1016/S1050-6411(02)00085-8

[4] W. H. Gage, D. A. Winter, J. S. Frank, and A. L. Adkin, "Kinematic and kinetic validity of the inverted pendulum model in quiet standing," Gait Posture, vol. 19, pp. 124-132, 2004.

https://doi.org/10.1016/S0966-6362(03)00037-7

[5] K. Masani, A. H. Vette, M. Kouzaki, H. Kanehisa, T. Fukunaga, and M. R. Popovic, "Larger center of pressure minus center of gravity in the elderly induces larger body acceleration during quiet standing," Neurosci Lett, vol. 422, pp. 202-206, 2007.

https://doi.org/10.1016/j.neulet.2007.06.019

[6] B. L. Day and M. J. Steiger, P. D. Thompson, and C. D. Marsden, "Effect of vision and stance width on human body motion when standing: implications for afferent control of lateral sway," J Physiol, vol. 469, pp. 479-499, 1993. https://doi.org/10.1113/jphysiol.1993.sp019824

[7] W. L. Hsu, J. P. Scholz, G. Schöner, J. J. Jeka, and T. Kiemel, "Control and estimation of posture during quiet stance depends on multijoint coordination," J Neurophysiol, vol. 97, pp. 3024-3035, 2007.

https://doi.org/10.1152/jn.01142.2006

[8] I. J. Pinter, R. van Swigchem, A. J. van Soest, and L. A. Rozendaal, "The dynamics of postural sway cannot be captured using a one-segment inverted pendulum model: a PCA on segment rotations during unperturbed stance," $J$ Neurophysiol, vol. 100, pp. 3197-3208, 2008.

https://doi.org/10.1152/jn.01312.2007

[9] M. Günther, S. Grimmer, T. Siebert, and R. Blickhan, "All leg joints contribute to quiet human stance: a mechanical analysis," J Biomech, vol. 42, pp. 2739-2746, 2009.

https://doi.org/10.1016/j.jbiomech.2009.08.014

[10] M. Günther, P. Putsche, L. Leistritz, and S. Grimmer, "Phase synchronisation of the three leg joints in quiet human stance," Gait Posture, vol. 33, pp. 412-417, 2011.

https://doi.org/10.1016/j.gaitpost.2010.12.014

[11] O. Caron, B. Faure, and Y. Brenière, "Estimating the centre of gravity of the body on the basis of the centre of pressure in standing posture," J Biomech, vol. 30, pp. 1169-1171, 1997.

https://doi.org/10.1016/S0021-9290(97)00094-8

[12] P. Rougier, C. Burdet, I. Farenc, and L. Berger, "Backward and forward leaning postures modelled by an $\mathrm{fBm}$ framework," Neurosci Res, vol. 41, pp. 41-50, 2001.

https://doi.org/10.1016/S0168-0102(01)00257-7

[13] T. E. Prieto, J. B. Myklebust, R. G. Hoffmann, E. G. Lovett, and B. M. Myklebust, "Measures of postural steadiness: differences between healthy young and elderly adults," IEEE Trans. Biomed Eng, vol. 43, pp. 956-966, 1996.

https://doi.org/10.1109/10.532130 
[14] V. Cornilleau-Pérès, N. Shabana, J. Droulez, J. C. Goh, G. S. Lee, and P. T. Chew, "Measurement of the visual contribution to postural steadiness from the COP movement: methodology and reliability," Gait Posture, vol. 22, pp. 96-106, 2005.

https://doi.org/10.1016/j.gaitpost.2004.07.009

[15] M. Kouzaki and K. Masani, "Postural sway during quiet standing is related to physiological tremor and muscle volume in young and elderly adults," Gait Posture, vol. 35, pp. 11-17, 2012.

https://doi.org/10.1016/j.gaitpost.2011.03.028

[16] N. A. Bernstein, The coordination and regulation of movements. London, Pergamon, 1967.

[17] R. Fitzpatrick, D. K. Rogers, and D. I. McCloskey, "Stable human standing with lower-limb muscle afferents providing the only sensory input," J Physiol, vol. 480, pp. 395-403, 1994.

https://doi.org/10.1113/jphysiol.1994.sp020369

[18] P. B. de Freitas, S. M. Freitas, M. Duarte, M. L. Latash, and V. M. Zatsiorsky, "Effects of jo int immobilization on standing balance," Hum Mov Sci, vol. 28, pp. 515-528, 2009.

https://doi.org/10.1016/j.humov.2009.02.001

[19] N. A. Kuznetsov and M. A. Riley, "Effects of breathing on multijoint control of center of mass position during upright stance," J Mot Behav, vol. 44, pp. 241-253, 2012.

https://doi.org/10.1080/00222895.2012.688894 